\title{
Zur grenzüberschreitenden Erbringung von Gesundheitsleistungen
}

\author{
Daniel Eryk Lach
}

A. Einleitende Bemerkungen - Terminologie

I. Grenzüberschreitende Gesundheitsversorgung und Definition der Sachleistungen

II. Begriffe des Versicherungsmitgliedstaates, des zuständigen Trägers und des zuständigen Mitgliedstaates

III. Versicherter und Patient

B. Das Recht auf Leistungen der Gesundheitsversorgung

I. Wohnort in einem anderen als dem zuständigen Mitgliedstaat 203

II. Grenzgänger

III. Aufenthalt außerhalb des zuständigen Mitgliedstaats

1. Die medizinische Notwendigkeit der Leistungen der Gesundheitsversorgung

2. Reisen zur Inanspruchnahme von Sachleistungen Die Genehmigung des Trägers

C. Patientenrechte in der grenzüberschreitenden Gesundheitsversorgung 208

I. Annahmen zur grenzüberschreitenden Gesundheitsversorgung 208

II. Zuständigkeiten der Mitgliedstaaten

1. Zuständigkeiten des Behandlungsmitgliedstaats

2. Zuständigkeiten des Versicherungsmitgliedstaats

III. Erstattung der Kosten

1. Allgemeine Grundsätze - Art. 7 der Richtlinie Nr. 2011/24/EU 214

2. Das Recht des Versicherungsmitgliedstaats auf die Beschränkung der Anwendung von Vorschriften über die Kostenerstattung

3. Das zur Kostenerstattung verpflichtete Subjekt

4. Gesundheitsversorgung, die einer Vorabgenehmigung unterliegen kann

a) Abschließender Katalog der Gesundheitsversorgung mit Vorabgenehmigung

b) Die Beschränkung der Zulässigkeit der Verweigerung der Vorabgenehmigung

D. Schlussbemerkungen 


\section{A. Einleitende Bemerkungen - Terminologie}

\section{Grenzüberschreitende Gesundheitsversorgung und Definition der Sachleistungen}

Beschäftigt man sich mit den gesetzlichen Regelungen zur grenzüberschreitenden Gesundheitsversorgung in der EU, sollte man zuerst kurz auf die in den gemeinschaftlichen Rechtsakten ${ }^{1}$ verwendete Terminologie eingehen und die rechtlichen Definitionen der Verordnungen und der Richtlinie vergleichen. Besonderes Augenmerk ist dabei auf die Definition der „grenzüberschreitenden Gesundheitsversorgung“ zu richten: In Art. 3 a der Richtlinie Nr. 2011/24/EU wird die „Gesundheitsversorgung“ als die Summe der Gesundheitsdienstleistungen definiert, die von Angehörigen der Gesundheitsberufe gegenüber Patienten erbracht werden, um deren Gesundheitszustand zu beurteilen, zu erhalten oder wiederherzustellen, einschließlich der Verschreibung, Abgabe und Bereitstellung von Arzneimitteln und Medizinprodukten. Nach Art. 3 e ist die „grenzüberschreitende Gesundheitsversorgung" eine Gesundheitsversorgung, die in einem anderen Mitgliedstaat als dem Versicherungsmitgliedstaat erbracht oder verschrieben wird.

Vor diesem Hintergrund sollte man Art. 1 va der Verordnung Nr. 883/2004 sehen, wonach Sachleistungen im Sinne von Titel III Kapitel 1 (Leistungen bei Krankheit sowie Leistungen bei Mutterschaft und gleichgestellte Leistungen bei Vaterschaft) solche Sachleistungen sind, „die nach den Rechtsvorschriften eines Mitgliedstaats vorgesehen sind und die den Zweck verfolgen, die ärztliche Behandlung und die diese Behandlung ergänzenden Produkte und Dienstleistungen zu erbringen bzw. zur Verfügung zu stellen oder direkt zu bezahlen oder die diesbezüglichen Kosten zu erstatten. Dazu gehören auch Sachleistungen bei Pflegebedürftigkeit.“ Im Lichte dieser Definition scheint der Begriff "Sachleistung“ eher rechtlichen Gehalt zu haben und die Aufgaben und Verpflichtungen des Trägers gegenüber dem Leistungsberechtigten zu beschreiben, wobei die Gesundheitsversorgung lediglich als Erbringung von Gesundheitsdienstleistungen im medizinischen Sinn verstanden werden soll. Man kann also sagen, dass die Sachleistungen im Sinne der Verordnung Nr. 883/2004 - als Anspruchsobjekt von Versicherten, d.h. Leistungsberechtigten des Gesundheitssystems) - die Zurverfügungstellung von Leistungen der Gesundheitsversorgung und/oder deren Bezahlung umfassen.

Dabei ist das Ziel der Sachleistungen zu berücksichtigen: Sie sollen nämlich „,den Zweck verfolgen, die ärztliche Behandlung und die diese Behandlung ergänzenden Produkte und Dienstleistungen zu erbringen bzw. zur Verfügung zu stellen oder direkt zu

1 Es geht hier um die Verordnung (EG) des Europäischen Parlaments und des Rates Nr. 883/2004 zur Koordinierung der Systeme der sozialen Sicherheit, die Verordnung (EG) des Europäischen Parlaments und des Rates Nr. 987/2009 zur Festlegung der Modalitäten für die Durchführung der Verordnung (EG) Nr. 883/2004 über die Koordinierung der Systeme der sozialen Sicherheit und die Richtlinie Nr. 2011/24/EU des Europäischen Parlaments und des Rates über die Ausübung der Patientenrechte in der grenzüberschreitenden Gesundheitsversorgung. 
bezahlen oder die diesbezüglichen Kosten zu erstatten“. Mit anderen Worten ist Gegenstand der Garantie die entsprechende ,ärztliche Behandlung“ samt „ergänzenden Produkten und Dienstleistungen“, wobei die Art und Weise der Realisierung dieser Garantie (Sicherung des Zugangs zur Gesundheitsversorgung, ihre „Direktzahlung“ oder „Kostenerstattung“) eher zweitrangig ist und von den in den einzelnen Mitgliedstaaten gewählten organisatorischen und finanziellen Lösungen abhängt. Die Verordnung Nr. 883/2004 betrifft lediglich die Koordinierung der Systeme der sozialen Sicherheit und nicht deren EU-weite Vereinheitlichung. Die Richtlinie (bzw. das diese implementierende nationale Recht) regelt dann nur die Kostenerstattungsfragen zwischen dem zuständigen Träger und dem Patienten (Leistungsberechtigten), die mit der Inanspruchnahme von Leistungen der grenzüberschreitenden Gesundheitsversorgung verbunden sind, und nicht die Rechtsverhältnisse zwischen den Trägern aus verschiedenen Mitgliedstaaten.

\section{Begriffe des Versicherungsmitgliedstaates, des zuständigen Trägers und des zuständigen Mitgliedstaates}

Laut Art. 3 c der Richtlinie Nr. 2011/24/EU ist Versicherungsmitgliedstaat derjenige Staat, der gemäß der Verordnung (EG) Nr. 883/2004 und der Verordnung (EG) Nr. 987/2009 dafür zuständig ist, dem Versicherten eine Vorabgenehmigung für die Inanspruchnahme angemessener Behandlungsleistungen außerhalb seines Wohnsitzmitgliedstaats zu erteilen. In Bezug auf Staatsangehörige eines Drittlands, die unter die Verordnung (EG) Nr. 859/2003 oder die Verordnung (EU) Nr. 1231/2010 fallen oder die die gesetzlichen Voraussetzungen des Versicherungsmitgliedstaats für einen Leistungsanspruch erfüllen, ist es der Staat, der dafür zuständig ist, dem Versicherten eine Vorabgenehmigung für die Inanspruchnahme angemessener Behandlungsleistungen in einem anderen Mitgliedstaat zu erteilen. Ist kein Mitgliedstaat gemäß jenen Verordnungen hierfür zuständig, so gilt als Versicherungsmitgliedstaat derjenige Mitgliedstaat, in dem der Betreffende versichert ist oder in dem er gemäß den Rechtsvorschriften dieses Mitgliedstaats einen Anspruch auf Leistungen bei Krankheit hat.

Nach Art. 1 der Verordnung Nr. 883/2004 ist der Mitgliedstaat zuständig, in dem der zuständige Träger seinen Sitz hat. Der Begriff „,zuständiger Träger“ bezeichnet die Institution, bei der die betreffende Person zum Zeitpunkt der Stellung des Antrags auf Leistungen versichert ist, oder die Institution, der gegenüber die betreffende Person einen Leistungsanspruch hat oder hätte, wenn sie selbst oder ihr Familienangehöriger bzw. ihre Familienangehörigen in dem Mitgliedstaat wohnen würden, in dem diese Institution ihren Sitz hat. Vorbehaltlich einer Reihe von Ausnahmen ist, wie im Fall der Verordnung Nr. 1408/71, das grundlegende Kriterium für die Bestimmung des ,zuständigen Trägers“" in Bezug auf die Beschäftigten der Mitgliedstaat der Ausübung der Beschäftigung - loci laboris - (Art. 11 Abs. 2a). 


\section{Versicherter und Patient}

Auch ist kurz das Verhältnis der Begriffe „Versicherter” und „Patient” zu erörtern. Nach Art. 3 b der Richtlinie Nr. 2011/24/EU sind Versicherte erstens die Personen (einschließlich ihrer Familienangehörigen und Hinterbliebenen), die unter Art. 2 der Verordnung (EG) Nr. 883/2004 fallen und die Versicherte im Sinne des Art. 1 c jener Verordnung sind, also alle Personen, die nach den Rechtsvorschriften des zuständigen Mitgliedstaats - unabhängig von der angewendeten Methode der sozialen Sicherung - die für einen Leistungsanspruch vorgesehenen Voraussetzungen erfüllen. Zweitens handelt es sich dabei um Staatsangehörige eines Drittlands, die unter die Verordnung (EG) Nr. 859/2003 oder die Verordnung (EU) Nr. 1231/2010 fallen oder die die gesetzlichen Voraussetzungen des Versicherungsmitgliedstaats für einen Anspruch auf Leistungen erfüllen. Patient ist demgegenüber nach Art. 3 h der Richtlinie Nr. 2011/24/EU jede natürliche Person, die Gesundheitsdienstleistungen in einem Mitgliedstaat in Anspruch nehmen möchte oder in Anspruch nimmt.

Bei Inanspruchnahme von Leistungen der Gesundheitsversorgung auf Kosten der zuständigen Systeme ist für die „Direktzahlung“ (oder „Kostenerstattung“) solcher Versorgung rechtlich relevant, ob die jeweilige Person zu den Leistungen berechtigt, also von der Systemgarantie umfasst war, nicht aber die diesbezüglichen Bedürfnisse und Wünsche dieser Person. Mit anderen Worten kommt es darauf an, ob diese Person als „versichert", d.h. als zu der Gesundheitsversorgung berechtigt definiert werden kann. $\mathrm{Zu}$ unterscheiden ist also der Begriff des Leistungsberechtigten im rechtlichen Sinne (als Versorgungs- bzw. Kostenerstattungsberechtigter) von dem des Leistungsempfängers im medizinischen Sinne (als Patient). Das wird gerade im Lichte der Vorschriften deutlich, die dem zuständigen Träger das Recht zur Erteilung der Vorabgenehmigung für die Nutzung der grenzüberschreitenden Gesundheitsversorgung im Sinne einer Versorgung außerhalb des zuständigen Mitgliedstaats) dem zuständigen Träger vorbehalten. So kann eine solche Genehmigung lediglich einem Leistungsberechtigten erteilt werden, der sich dann auf ihrer Grundlage in einen anderen Mitgliedstaat begeben kann, um auf Rechnung des zuständigen Trägers eine seinem Zustand angemessene Behandlung zu erhalten. Erst dann wird er zum Patienten. Demgegenüber können die Patienten, die nach dem anzuwendenden Recht nicht zu den Leistungen (bzw. deren Finanzierung) im Rahmen des Systems der Gesundheitsversorgung berechtigt sind, zwar unbeschränkt sowohl inländische als auch grenzüberschreitende Gesundheitsversorgung genießen, ihre Entscheidungen in dieser Angelegenheit sind jedoch im Hinblick auf die Regelungen über die Kostenerstattung für Gesundheitsversorgungsleistungen irrelevant, weil die Vorschriften nur für die zur Gesundheitsversorgung Berechtigten gelten, d.h. für Personen, die von der Systemgarantie umfasst sind. 


\section{B. Das Recht auf Leistungen der Gesundheitsversorgung}

\section{Wohnort in einem anderen als dem zuständigen Mitgliedstaat}

Nach Art. 17 der Verordnung Nr. 883/2004 erhalten Versicherte (bzw. ihre Familienangehörigen), die in einem anderen als dem zuständigen Mitgliedstaat wohnen, in dem Wohnmitgliedstaat Sachleistungen, die vom Träger des Wohnorts nach den für ihn geltenden Rechtsvorschriften für Rechnung des zuständigen Trägers erbracht werden, als ob sie nach diesen Rechtsvorschriften versichert wären. Die Vorschrift des Art. 17 bezieht sich also auf eine Situation, in der der Versicherte ständig in einem Mitgliedstaat eine Beschäftigung oder selbstständige Erwerbstätigkeit ausübt, wobei sein Wohnort sich in einem anderen Mitgliedstaat befindet. Die Sachleistungen werden ihm dann vom Träger des Wohnorts erbracht, aber auf Kosten des ,zuständigen Trägers“.

Gemäß Art. 24 der Umsetzungsverordnung Nr. 987/2009 müssen sich der Versicherte und/oder seine Familienangehörigen dazu beim Träger ihres Wohnorts eintragen lassen. Ihr Sachleistungsanspruch im Wohnmitgliedstaat wird dann durch ein Dokument bescheinigt, das vom zuständigen Träger auf Antrag des Versicherten oder auf Antrag des Trägers des Wohnorts ausgestellt wird. Von praktischer Bedeutung ist, dass das genannte Dokument solange gilt, bis der zuständige Träger den Träger des Wohnorts über seinen Widerruf informiert. Sonst ist der Träger des Wohnorts verpflichtet, den zuständigen Träger von jeder Eintragung und von jeder Änderung oder Streichung dieser Eintragung zu benachrichtigen.

\section{Grenzgänger}

Art. 18 der Verordnung Nr. 883/2004 enthält Sonderregeln für die Grenzgänger ${ }^{2}$ (und ihre Familienangehörigen) und zwar für den Fall des Aufenthalts in dem zuständigen Mitgliedstaat, wenn sich der Wohnort in einem anderen Mitgliedstaat befindet. Nach Abs. 1 haben die in Art. 17 genannten Versicherten auch während des Aufenthalts in dem zuständigen Mitgliedstaat Anspruch auf Sachleistungen. Die Sachleistungen werden vom zuständigen Träger auf dessen Rechnung nach den für ihn geltenden Rechtsvorschriften erbracht, als ob die betreffenden Personen in diesem Mitgliedstaat wohnten.

Die Familienangehörigen von Grenzgängern haben nur dann Anspruch auf Sachleistungen während ihres Aufenthalts in dem zuständigen Mitgliedstaat, wenn dieser Mit-

2 Nach Art. 1 f der Verordnung Nr. 883/2004 ist ein „Grenzgänger“ eine Person, die in einem Mitgliedstaat eine Beschäftigung oder eine selbstständige Erwerbstätigkeit ausübt und in einem anderen Mitgliedstaat wohnt, in den sie in der Regel täglich, mindestens jedoch einmal wöchentlich zurückkehrt. 
gliedstaat in Anhang III der Verordnung nicht aufgeführt worden ist. ${ }^{3}$ In diesem Fall haben die Familienangehörigen in dem zuständigen Mitgliedstaat Anspruch auf Sachleistungen unter den Voraussetzungen des Art. 19 Abs. 1, die den Aufenthalt außerhalb des zuständigen Mitgliedstaats betreffen.

\section{Aufenthalt außerhalb des zuständigen Mitgliedstaats}

\section{Die medizinische Notwendigkeit der Leistungen der Gesundheitsversorgung}

Das Recht auf Leistungen der Gesundheitsversorgung während des Aufenthalts außerhalb des zuständigen Mitgliedstaats ist in Art. 19 der Verordnung Nr. 883/2004 geregelt. Art. 19 Abs. 1 nennt als objektives und offensichtliches Kriterium die medizinische Notwendigkeit der Leistung, die während des Aufenthalts des Versicherten und seiner Familienangehörigen außerhalb des zuständigen Mitgliedstaats auftreten muss. Auch die Art der Leistungen und die voraussichtliche Dauer des Aufenthalts ist zu berücksichtigen. Falls die genannten Voraussetzungen erfüllt sind, werden die entsprechenden Leistungen vom Träger des Aufenthaltsorts nach den für ihn geltenden Rechtsvorschriften auf Rechnung des zuständigen Trägers erbracht, als ob die betreffenden Personen nach diesen Rechtsvorschriften versichert wären.

Detaillierte Umsetzungsvorschriften beinhaltet Art. 25 der Verordnung Nr. 987/2009, der das Verfahren und den Umfang des Anspruchs (Abs. 1-3) sowie das Verfahren und die Modalitäten der Übernahme und/oder Erstattung von Sachleistungen (Abs. 4-9) regelt.

Gemäß Art. 25 Abs. 1 der Verordnung Nr. 987/2009 legt bei Anwendung von Art. 19 der Verordnung Nr. 883/2004 der Versicherte dem Erbringer von Gesundheitsleistungen im Aufenthaltsmitgliedstaat ein von dem zuständigen Träger ausgestelltes Dokument vor, das seinen Sachleistungsanspruch bescheinigt. Verfügt der Versicherte nicht über ein solches Dokument, so fordert der Träger des Aufenthaltsorts auf Antrag oder falls andernfalls erforderlich das Dokument beim zuständigen Träger an. Dieses Dokument bescheinigt, dass der Versicherte unter den Voraussetzungen des Art. 19 der Verordnung Nr. 883/2004 zu denselben Bedingungen wie die nach den Rechtsvorschriften des Aufenthaltsmitgliedstaats versicherten Personen Anspruch auf Sachleistungen hat.

Praktisch relevant ist die Vorschrift des Art. 25 Abs. 3 der Verordnung Nr. 987/2009, der präzisiert, dass die Sachleistungen im Sinne von Art. 19 Abs. 1 der Verordnung Nr. 883/2004 diejenigen sind, die im Aufenthaltsmitgliedstaat nach dessen Rechtsvorschriften erbracht werden und sich als medizinisch notwendig erweisen, damit der Versicher-

3 Im diesem Anhang sind folgende Mitgliedstaaten genannt: Dänemark, Irland, Finnland, Schweden, Vereinigtes Königreich sowie Estland, Spanien, Litauen, Italien, Ungarn und Niederlande. Die Einträge, die die zweite Gruppe der in Anhang III genannten Staaten betreffen, treten vier Jahre nach dem Geltungsbeginn der Verordnung außer Kraft (Art. 87 Abs. 10a der Verordnung Nr. 883/2004). 
te nicht vorzeitig in den zuständigen Mitgliedstaat zurückkehren muss, um die erforderlichen medizinischen Leistungen zu erhalten. Dies bedeutet, dass in jedem Einzelfall, nicht nur die Gesundheit des Versicherten, sondern auch die Umstände seines Aufenthalts außerhalb des zuständigen Staates (die Dauer des Aufenthalts und sein geplantes Ende) bewertet werden sollen. In der Tat sind in erster Linie die Pläne des Versicherten von Bedeutung, weil in ihrem Kontext geprüft wird, ob die Gesundheitsversorgung durch den Staat des Aufenthalts geleistet werden sollte, oder ob man „warten“ kann, bis der Versicherte in den zuständigen Staat zurückkehrt. Ziel ist nämlich die „Verhinderung der vorzeitigen Rückkehr“. In der Praxis muss der Versicherte zumindest glaubhaft machen, dass ein konkretes Datum, das er als „Ende des geplanten Aufenthalts“ nennt, irgendwie gerechtfertigt und begründet ist.

Das in Art. 19 vorgesehene Recht auf medizinisch notwendige Leistungen der Gesundheitsversorgung hat also mit der allgemeinen Verpflichtung jedes Angehörigen der Gesundheitsberufe, medizinische Hilfe im Fall der Bedrohung des Lebens oder der Gesundheit zu leisten, nichts zu tun. Die Leistungsanspruchsvoraussetzung aus Art. 19 der Verordnung Nr. 883/2004 ist die Notwendigkeit der Versorgung, die im Lichte der Pläne des Berechtigten beurteilt wird, und nicht die objektive Bedrohung seines Lebens. Die Regelung für Notfälle ist in den Vorschriften der Umsetzungsverordnung enthalten, die die geplante Behandlung außerhalb des zuständigen Systems betreffen (Art. 26 Abs. 3 der Verordnung Nr. 987/2009 - s.u.).

Wenn es um die Verfahren und Bestimmungen der Aufbringung und der Erstattung der Kosten von Sachleistungen geht, finden die Vorschriften von Art. 25 Abs. 4-9 der Verordnung Nr. 987/2009 Anwendung. Danach kann der Versicherte die Erstattung beim Träger des Aufenthaltsorts beantragen, wenn er die Kosten aller oder eines Teils der im Rahmen von Art. 19 der Verordnung Nr. 883/2004 erbrachten Sachleistungen selbst getragen hat und die vom Träger des Aufenthaltsorts angewandten Rechtsvorschriften es ermöglichen, dass diese Kosten dem Versicherten erstattet werden. In diesem Fall erstattet dieser dem Leistungsberechtigten direkt den diesen Leistungen entsprechenden Betrag innerhalb der Grenzen und Bedingungen der nach seinen Rechtsvorschriften geltenden Erstattungssätze. Falls aber die Erstattung dieser Kosten nicht unmittelbar beim Träger des Aufenthaltsorts beantragt wurde, so werden diese vom zuständigen Träger nach den für den Träger des Aufenthaltsorts geltenden Erstattungssätzen oder den Beträgen erstattet, die dem Träger des Aufenthaltsortes erstattet worden wären. Der Träger des Aufenthaltsorts ist verpflichtet, dem zuständigen Träger auf dessen Ersuchen die erforderlichen Auskünfte über diese Erstattungssätze oder Beträge zu erteilen. Der zuständige Träger kann die entstandenen Kosten innerhalb der Grenzen und nach Maßgabe der in seinen Rechtvorschriften niedergelegten Erstattungssätze erstatten, aber nur sofern sich der Versicherte mit der Anwendung dieser Bestimmung einverstanden erklärt hat. Für den Fall, dass die Rechtsvorschriften des Aufenthaltsmitgliedstaats in dem betreffenden Fall keine Erstattung nach den Abs. 4 und 5 vorsehen, kann der zuständige Träger laut Abs. 7 die Kosten innerhalb der Grenzen und nach 
Maßgabe der in seinen Rechtvorschriften festgelegten Erstattungssätze erstatten, ohne dass das Einverständnis des Versicherten erforderlich wäre. Die Erstattung an den Versicherten kann in keinem Fall den Betrag der ihm tatsächlich entstandenen Kosten überschreiten. Von praktischer Bedeutung kann sein, dass der zuständige Träger dem Versicherten im Fall erheblicher Ausgaben einen angemessenen Vorschuss zahlen kann, nachdem dieser den Erstattungsantrag bei ihm eingereicht hat.

Relevant ist auch die Regelung in Art. 19 Abs. 2 der Verordnung Nr. 883/2004, wonach die bei der Kommission der Europäischen Gemeinschaften eingesetzte Verwaltungskommission 4 eine Liste der Sachleistungen erstellt, für die aus praktischen Gründen eine vorherige Vereinbarung zwischen der betreffenden Person und dem die medizinische Leistung erbringenden Träger erforderlich ist, damit diese Sachleistungen während eines Aufenthalts in einem anderen Mitgliedstaat erbracht werden können.

\section{Reisen zur Inanspruchnahme von Sachleistungen - Die Genehmigung des Trägers}

Wie aus der Rechtsprechung des EuGH ${ }^{5}$ folgt, sind in der Praxis jedoch die Probleme von größter Bedeutung, die mit der grundsätzlich geplanten Inanspruchnahme von Leistungen der Gesundheitsversorgung außerhalb des zuständigen Mitgliedstaats verbunden sind. Diese Fragen sind aktuell in Art. 20 der Verordnung Nr. 883/2004 geregelt. Demgemäß muss ein Versicherter, der sich zur Inanspruchnahme von Sachleistungen in einen anderen Mitgliedstaat begibt, die Genehmigung des zuständigen Trägers einholen. Erst wenn eine solche Genehmigung erteilt wurde, erhält er die Sachleistungen, die vom Träger des Aufenthaltsorts nach den für ihn geltenden Rechtsvorschriften auf Rechnung des zuständigen Trägers erbracht werden, als ob er nach diesen Rechtsvorschriften versichert wäre. Zusätzlich nennt Art. 20 Abs. 2 zwei Voraussetzungen, von deren Erfüllung die Erteilung der Genehmigung abhängig ist. Erstens muss die betreffende Behandlung Teil der Leistungen sein, die nach den Rechtsvorschriften des Wohnmitgliedstaats der betreffenden Person vorgesehen sind. Zweitens muss der Fall vorliegen, dass diese Behandlung nicht innerhalb eines in Anbetracht des derzeitigen Gesundheitszustands des Berechtigten und des voraussichtlichen Verlaufs seiner Krankheit medizinisch vertretbaren Zeitraums gewährt werden kann. Das gleiche gilt entsprechend für die Familienangehörigen des Versicherten (Art. 20 Abs. 3 der Verordnung Nr. 883/2004).

4 Die Zusammensetzung und Arbeitsweise der Verwaltungskommission regelt Art. 71; deren Aufgaben Art. 72 der Verordnung Nr. 883/2004.

5 Vgl. EuGH v. 28.4.1998, Rs. C-158/96 (Kohll), Slg. 1998, S. I-1931; EuGH v. 28.4.1998, Rs. C120/95 (Decker), Slg. 1998, S. I-1831; EuGH v. 12.7.2001, Rs. C-157/99 (Smits und Peerbooms), Slg. 2001, S. I-5473; EuGH v. 12.7.2001, Rs. C-368/98 (Vanbraekel u.a.), Slg. 2001, S. I-5363; EuGH v. 13.5.2003, Rs. C-385/99 (Müller-Fauré und van Riet), Slg. 2003, S. I-4509; EuGH v. 23.10.2003, Rs. C-56/01 (Inizan), Slg. 2003, S. I-12403; EuGH v. 18.3.2004, Rs. C-8/02 (Leichtle), Slg. 2004, S. I-2461; EuGH v. 16.5.2006, Rs. C-372/04 (Watts), Slg. 2006, S. I-4325. 
Eine bemerkenswerte Änderung hinsichtlich der Regelung der Verordnung Nr. 1408/71 ist die Ersetzung der Formulierung „Die [...] Genehmigung darf nicht verweigert werden“ durch die Anordnung, dass „die Genehmigung [...] erteilt [wird]“, wenn die oben genannten Kriterien 6 erfüllt sind. Das ungeschickt formulierte Verbot der Verweigerung der Genehmigung, was darauf hindeuten könnte, dass die Genehmigung nur in Ausnahmefällen erteilt werden solle, wurde durch eine klare Pflicht ersetzt, was jedoch nichts an der Tatsache ändert, dass nach wie vor die Bewertung, ob die betreffende Behandlung ,innerhalb einer aus medizinischer Sicht angemessenen Zeit" gewährt werden kann, als ein Streitpunkt bleibt. Der EuGH hat sich bereits mehrmals mit dieser Frage (aufgrund des Art. 22 der Verordnung Nr. 1408/71) in Bezug auf die Wartelisten und auf sonstige Instrumente der Rationierung des Zugangs zu Leistungen der Gesundheitsversorgung in ausgewählten Systemen auseinandergesetzt.

Umsetzungsvorschriften sind in Art. 26 der Verordnung Nr. 987/2009 enthalten. Nach Abs. 1 legt der Versicherte im Fall der geplanten Inanspruchnahme von Sachleistungen außerhalb des zuständigen Systems(Staats) dem Träger des Aufenthaltsorts ein vom zuständigen Träger - also dem, der die Kosten der geplanten Behandlung zu tragen hat - ausgestelltes Dokument vor. Wohnt der Versicherte nicht in dem zuständigen Mitgliedstaat, so muss er die Genehmigung beim Träger des Wohnorts beantragen, der den Antrag dann unverzüglich an den zuständigen Träger weiterleitet. In diesem Fall bescheinigt der Träger des Wohnorts, ob die Bedingungen des Art. 20 Abs. 2 S. 2 der Verordnung Nr. 883/2004 in dem Wohnmitgliedstaat erfüllt sind.

Wie schon erwähnt (und wie auch unmissverständlich aus der Rechtsprechung des EuGH folgt), kann der zuständige Träger die beantragte Genehmigung nur dann verweigern, wenn nach Einschätzung des Trägers des Wohnorts die Bedingungen des Art. 20 Abs. 2 S. 2 der Verordnung Nr. 883/2004 in dem Wohnmitgliedstaat des Versicherten nicht erfüllt sind oder wenn die gleiche Behandlung im zuständigen Mitgliedstaat selbst innerhalb eines in Anbetracht des derzeitigen Gesundheitszustands und des voraussichtlichen Verlaufs der Krankheit medizinisch vertretbaren Zeitraums gewährt werden kann. Der zuständige Träger ist verpflichtet, dem Träger des Wohnortes seine Entscheidung mitzuteilen. Geht innerhalb der nach innerstaatlichem Recht des betreffenden Mitgliedstaats geltenden Fristen keine Antwort ein, so gilt die Genehmigung als durch den zuständigen Träger erteilt, was für den Berechtigten günstig ist.

Die Notfälle regelt Art. 26 Abs. 3 der Verordnung Nr. 987/2009, wonach der Träger des Wohnorts die Genehmigung auf Rechnung des zuständigen Trägers erteilt und den zuständigen Träger unverzüglich hiervon unterrichtet, wenn eine versicherte Person, die nicht in dem zuständigen Mitgliedstaat wohnt, eine dringende und lebensnotwendige Behandlung benötigt und die Genehmigung nach Art. 20 Abs. 2 S. 2 der Verordnung

6 D.h., die betreffende Behandlung ist Teil der Leistungen, die nach den Rechtsvorschriften des Wohnmitgliedstaats der betreffenden Person vorgesehen sind, und diese Behandlung kann nicht innerhalb eines in Anbetracht ihres derzeitigen Gesundheitszustands und des voraussichtlichen Verlaufs ihrer Krankheit medizinisch vertretbaren Zeitraums gewährt werden. 
Nr. 883/2004 nicht verweigert werden darf. Laut Satz 2 dieses Absatzes akzeptiert der zuständige Träger die Befunde und therapeutischen Entscheidungen der vom Träger des Wohnorts autorisierten Ärzte bezüglich der Erforderlichkeit einer dringenden lebensnotwendigen Behandlung. Dies sollte wohl so verstanden werden, dass der zuständige Träger verpflichtet ist, die medizinischen Bewertungen bezüglich der Existenz des Notstandes anzuerkennen und diese nicht in Frage zu stellen, was zur Ablehnung des Kostenerstattungsantrags führen könnte. Diese Interpretation ist auch im Lichte des Art. 26 Abs. 4 der Verordnung Nr. 883/2004 berechtigt. Demgemäß behält der zuständige Träger das Recht, den Versicherten jederzeit im Verlauf des Genehmigungsverfahrens von einem Arzt seiner Wahl im Aufenthalts- oder Wohnmitgliedstaat untersuchen zu lassen. Die Vorschrift des Abs. 3 ist demgegenüber als eine Sonderregelung zu sehen, sie betrifft jedoch nicht das Genehmigungsverfahren, sondern einen Notstand, wenn eine dringende und lebensnotwendige Behandlung erteilt werden muss. In einer solchen Situation darf der zuständige Träger nicht die ihm in Art. 26 Abs. 4 vorbehaltenen Rechte nutzen.

Die Problematik der Übernahme der dem Versicherten entstandenen Kosten für Sachleistungen ist in Art. 26 Abs. 6-7 geregelt. Die vom EuGH bereits entschiedenen praktischen Fragen berücksichtigend hat der europäische Rechtsgeber auch die Übernahme der Reise- und Aufenthaltskosten bei geplanten Behandlungen vorgesehen (Art. 26 Abs. 8). Der zuständige Träger ist zur Erstattung der mit der Behandlung des Versicherten untrennbar verbundenen Reise- und Aufenthaltskosten verpflichtet, aber nur in den Fällen, in denen es von den nationalen Rechtsvorschriften des zuständigen Trägers so vorgesehen wurde.

\section{Patientenrechte in der grenzüberschreitenden Gesundheitsversorgung}

\section{Annahmen zur grenzüberschreitenden Gesundheitsversorgung}

Bereits in der 64 Absätze umfassenden Präambel präsentiert der EU-Rechtsgeber die maßgeblichen Thesen und Annahmen der Richtlinie Nr. 2011/24/EU. Obwohl sie keinen normativen Charakter haben, sind sie bei der Interpretation der Bestimmungen der Richtlinie und des diese implementierenden nationalen Rechts hilfreich.

Die Präambel betont vor allem, dass die Patienten zwar auf der Grundlage der Richtlinie grenzüberschreitende Gesundheitsdienstleistungen in Anspruch nehmen könnten, doch seien die Mitgliedstaaten nach wie vor für die Bereitstellung sicherer, hochwertiger und effizienter Gesundheitsdienstleistungen in ausreichendem Umfang für die Bürger in ihrem Hoheitsgebiet verantwortlich. Zudem sollten Patienten bei der Umsetzung der Richtlinie in einzelstaatliche Rechtsvorschriften und bei deren Anwendung nicht dazu ermuntert werden, Behandlungen in einem anderen als ihrem Versicherungsmit- 
gliedstaat in Anspruch zu nehmen. Der EU-Rechtsgeber weist auch wie in der Präambel der Verordnung Nr. 883/2004 auf die Rechtsprechung des EuGH hin, die die Inanspruchnahme der grenzüberschreitenden Gesundheitsversorgung betreffe, vor allem die Kostenerstattung für Gesundheitsdienstleistungen, die in einem anderen Mitgliedstaat als demjenigen, in dem der Empfänger der Behandlungsleistung seinen Wohnsitz habe, erbracht würden. Dabei verleiht er der Hoffnung Ausdruck, dass mit der Richtlinie eine allgemeinere und auch wirksame Anwendung der Grundsätze erreicht werde, die der Gerichtshof in Einzelfällen entwickelt habe. In Abs. 10 wird diese Aussage noch verstärkt: So ziele die Richtlinie darauf ab, Regeln zu schaffen, die den Zugang zu einer sicheren und hochwertigen grenzüberschreitenden Gesundheitsversorgung in der Union erleichtern, die Patientenmobilität im Einklang mit den vom Gerichtshof aufgestellten Grundsätzen gewährleisten und die Zusammenarbeit der Mitgliedstaaten bei der Gesundheitsversorgung fördern würden, wobei gleichzeitig die Zuständigkeiten der Mitgliedstaaten für die Festlegung der gesundheitsbezogenen Sozialversicherungsleistungen und für die Organisation und die Bereitstellung von Gesundheitsdienstleistungen und medizinischer Versorgung sowie der Sozialversicherungsleistungen, insbesondere im Krankheitsfall, uneingeschränkt beachtet werden sollten. Gleichzeitig wird festgehalten, dass weder die besondere Art noch die Organisation oder Finanzierung der Gesundheitsversorgung dazu führten, dass Gesundheitsdienstleistungen nicht unter den elementaren Grundsatz der Dienstleistungsfreiheit fielen. Nichtsdestotrotz könne sich der Versicherungsmitgliedstaat aus Gründen, die in der Qualität und Sicherheit der erbrachten Gesundheitsdienstleistung lägen, dafür entscheiden, die Kostenerstattung für die grenzüberschreitende Gesundheitsversorgung zu begrenzen, wenn sich dies durch zwingende Gründe des Allgemeininteresses bezogen auf die öffentliche Gesundheit rechtfertigen lasse.

Als selbstverständlich gilt, dass die Verpflichtung zur Kostenerstattung für die grenzüberschreitende Gesundheitsversorgung eindeutig auf Gesundheitsdienstleistungen beschränkt sein solle, auf die der Versicherte nach den Rechtsvorschriften des Versicherungsmitgliedstaats Anspruch habe, mit dem Vorbehalt, dass dem Patienten die Übernahme der Kosten für diese Gesundheitsdienstleistungen mindestens auf demselben Niveau garantiert werden solle, wie sie bei einer Versorgung im Versicherungsmitgliedstaat gewährt worden wäre. Abs. 32 betont, dass die Patienten in keinem Fall einen finanziellen Vorteil aus der in einem anderen Mitgliedstaat geleisteten Gesundheitsversorgung ziehen sollten; die Kostenübernahme solle daher auf die tatsächlichen Kosten der empfangenen Gesundheitsdienstleistungen begrenzt werden.

Die Präambel weist auch darauf hin, dass die Richtlinie nicht für jene Dienstleistungen der Langzeitpflege gelte, und dass der Zugang zu Organen und deren Zuteilung zum Zweck der Organtransplantation angesichts ihrer Besonderheit nicht in den Geltungsbereich dieser Richtlinie fallen solle. Bezugnehmend auf den Umfang der garantierten grenzüberschreitenden Gesundheitsversorgung wird auch festgestellt, dass die Richtlinie nicht nur für den Fall gelten solle, dass der Patient eine Gesundheitsbehandlung in 
einem anderen als seinem Versicherungsmitgliedstaat erhalte, sondern auch für die Verschreibung, Abgabe und Bereitstellung von Arzneimitteln und Medizinprodukten, wenn diese im Zusammenhang mit dieser Gesundheitsdienstleistung erfolgten. Der Begriff der grenzüberschreitenden Gesundheitsversorgung solle sowohl den Fall umfassen, dass ein Patient solche Arzneimittel und Medizinprodukte in einem anderen als seinem Versicherungsmitgliedstaat kaufe, als auch den Fall, dass er sie in einem anderen Mitgliedstaat kaufe als dem, in dem die Verschreibung ausgestellt worden sei.

Soweit es aber um den persönlichen Geltungsbereich der Richtlinie geht, postuliert Abs. 18, dass die Mitgliedstaaten in ihren nationalen Rechtsvorschriften weiterhin festlegen können sollten, wer im Rahmen der gesetzlichen Bestimmungen über das öffentliche Gesundheitswesen und die Sozialversicherung als Versicherter betrachtet werde, solange die in dieser Richtlinie festgelegten Patientenrechte gewährleistet seien. Gleichzeitig wird betont, dass die Mitgliedstaaten auch sicherstellen sollten, dass solche Werte wie Universalität, Zugang zu qualitativ hochwertiger Versorgung, Gleichbehandlung und Solidarität im Hinblick auf Patienten und Bürger aus anderen Mitgliedstaaten gewahrt würden und dass alle Patienten gleich behandelt würden, und zwar in Abhängigkeit von ihrem Bedarf an Gesundheitsdienstleistungen und nicht davon, in welchem Mitgliedstaat sie versichert seien. Dabei sollten die Mitgliedstaaten die Grundsätze der Freizügigkeit im Binnenmarkt, der Nichtdiskriminierung unter anderem aufgrund der Staatsangehörigkeit sowie der Erforderlichkeit und der Angemessenheit jeglicher Einschränkungen der Freizügigkeit achten. Klargestellt wird aber auch, dass die Richtlinie die Gesundheitsdienstleister nicht verpflichten solle, Patienten aus anderen Mitgliedstaaten für eine geplante Behandlung zu akzeptieren oder bevorzugt zu behandeln, wenn sich dadurch Nachteile für andere Patienten ergäben, etwa durch längere Wartezeiten für deren Behandlung. Der Zustrom von Patienten könne zu einer Nachfragesituation führen, die die in einem Mitgliedstaat bestehenden Kapazitäten für eine bestimmte Behandlung übersteige. In solchen Ausnahmefällen solle der Mitgliedstaat die Möglichkeit behalten, im Einklang mit den Art. 52 und 62 AEUV aus Gründen der öffentlichen Gesundheit Abhilfe zu schaffen. Diese Einschränkung solle jedoch nicht die Verpflichtungen der Mitgliedstaaten nach der Verordnung Nr. 883/2004 berühren.

Eindeutig festzuhalten ist, dass die Richtlinie nicht die Rechte eines Versicherten nach dieser Verordnung oder nach anderen Verordnungen berühren solle, die die Koordinierung der Sozialversicherungssysteme regelten. ${ }^{7}$ Für Patienten müssten beide Sys-

7 Dies sind, außer der Verordnung Nr. 883/2004: die Verordnung (EWG) Nr. 1408/71 des Rates vom 14. Juni 1971 zur Anwendung der Systeme der sozialen Sicherheit auf Arbeitnehmer und Selbständige sowie deren Familienangehörige, die innerhalb der Gemeinschaft zu- und abwandern, die Verordnung (EU) Nr. 1231/2010 des Europäischen Parlaments und des Rates vom 24. November 2010 zur Ausdehnung der Verordnung (EG) Nr. 883/2004 und der Verordnung (EG) Nr. 987/2009 auf Drittstaatsangehörige, die ausschließlich aufgrund ihrer Staatsangehörigkeit nicht bereits unter diese Verordnungen fallen, und die Verordnung (EG) Nr. 859/2003 des Rates vom 14. Mai 2003 zur Ausdehnung der Bestimmungen der Verordnung (EWG) Nr. 1408/71 und der Verordnung (EWG) 
teme nämlich kohärent sein: Entweder die vorliegende Richtlinie oder aber die Verordnungen der Union zur Koordinierung der Sozialversicherungssysteme gelangten zur Anwendung. Die Patienten sollten also nicht die ihnen vorteilhafteren Ansprüche gemäß den Verordnungen der Union zur Koordinierung der Sozialversicherungssysteme verlieren, wenn die Bedingungen erfüllt seien, was insbesondere heißt, dass jeder Patient, der eine Vorabgenehmigung für eine auf seinen Gesundheitszustand abgestimmte Behandlung in einem anderen Mitgliedstaat beantrage, stets diese Genehmigung nach Maßgabe der Bedingungen der Verordnungen der Union erhalten solle. Auch wird unterstrichen, dass die Richtlinie weder die Übertragung von Sozialversicherungsansprüchen zwischen den Mitgliedstaaten noch eine anderweitige Koordinierung der Sozialversicherungssysteme vorsehen solle. Einziges Ziel der Bestimmungen über Vorabgenehmigung und Kostenerstattung für die in anderen Mitgliedstaaten erbrachten Gesundheitsdienstleistungen solle es sein, die Freizügigkeit der Patienten zur Inanspruchnahme von Gesundheitsdienstleistungen zu gewährleisten und ungerechtfertigte Hindernisse für diese Grundfreiheit in den Versicherungsmitgliedstaaten der Patienten zu beseitigen.

Vor diesem Hintergrund sollte man noch Abs. 46 erwähnen, wonach die Genehmigung auch in dem Fall erteilt und die Leistungen gemäß der Verordnung (EG) Nr. 883/2004 gewährt werden sollten, dass ein Mitgliedstaat beschließe, ein System der Vorabgenehmigung für die Übernahme der Kosten einer Krankenhaus- oder Spezialbehandlung in einem anderen Mitgliedstaat nach Maßgabe der Richtlinie einzuführen, sofern die Bedingungen der Verordnung (EWG) Nr. 1408/71 oder der Verordnung (EG) Nr. 883/2004 erfüllt seien, es sei denn, der Patient habe etwas anderes beantragt. Dies solle insbesondere in Fällen gelten, in denen die Genehmigung nach einer administrativen oder gerichtlichen Überprüfung des Antrags erteilt werde und die betreffende Person die Behandlung in einem anderen Mitgliedstaat erhalten habe. In diesem Fall sollten Art. 7 und 8 der vorliegenden Richtlinie nicht gelten.

Außerdem wird darauf hingewiesen, dass nach der Rechtsprechung des Gerichtshofs eine Beschränkung der Dienstleistungsfreiheit vorliege, wenn die Übernahme der Kosten für in einem anderen Mitgliedstaat erbrachte Gesundheitsdienstleistungen durch das gesetzliche Sozialversicherungssystem oder das nationale Gesundheitssystem von einer Vorabgenehmigung abhängig gemacht werde. Gleichzeitig aber wird betont, dass die Patientenströme zwischen den Mitgliedstaaten beschränkt seien und es voraussichtlich auch bleiben würden, da die große Mehrheit der Patienten in der Union Gesundheitsdienstleistungen in ihrem eigenen Land in Anspruch nehme und dies vorziehe. Unter bestimmten Umständen könnten Patienten jedoch die Inanspruchnahme gewisser Formen der Gesundheitsversorgung in einem anderen Mitgliedstaat anstreben. Als Beispiel werden Gesundheitsdienstleistungen in Grenzgebieten genannt, für die die nächstgelegene geeignete Einrichtung jenseits der Grenze liege. Außerdem wollten manche Patienten im Ausland behandelt werden, um in der Nähe ihrer Angehörigen zu sein, die in

Nr. 574/72 auf Drittstaatsangehörige, die ausschließlich aufgrund ihrer Staatsangehörigkeit nicht bereits unter diese Bestimmungen fallen. 
einem anderen Mitgliedstaat lebten, oder um Zugang zu einer anderen Behandlungsmethode zu haben, die im Versicherungsmitgliedstaat nicht angeboten werde, oder weil sie glaubten, dass die Gesundheitsversorgung in einem anderen Mitgliedstaat qualitativ besser sei. In diesem Kontext wird die Anwendung der Vorabgenehmigung in Bezug auf Krankenhausbehandlungen als gerechtfertigt bewertet. Begründet wird dies damit, dass die Zahl der Krankenhäuser, ihre geografische Verteilung, ihr Ausbau und die Einrichtungen, über die sie verfügten, oder auch die Art der medizinischen Leistungen, die sie anbieten könnten, Faktoren seien, deren Planung, die grundsätzlich auf die Befriedigung vielfältiger Bedürfnisse ausgerichtet sei, möglich sein müsse. Diese Planung bezwecke, dass im betreffenden Mitgliedstaat ein ausgewogenes Angebot von qualitativ hochwertigen Krankenhausversorgungsleistungen ständig in ausreichendem $\mathrm{Ma} ß \mathrm{zu}-$ gänglich sei. Außerdem solle die Planung dazu beitragen, die Kosten zu beherrschen und soweit wie möglich jede Verschwendung finanzieller, technischer und personeller Ressourcen zu verhindern. Nach Abs. 41 gilt die gleiche Argumentation auch für diejenige ambulante Gesundheitsversorgung, die den Einsatz einer hoch spezialisierten und kostenintensiven medizinischen Infrastruktur oder Ausrüstung erfordert.

Bezugnehmend auf die in ständiger Rechtsprechung des EuGH festgestellten Kriterien für die Erteilung oder Verweigerung einer Vorabgenehmigung und deren Begrenzung auf das, was angesichts zwingender Gründe des Allgemeininteresses notwendig und angemessen ist, wird darauf hingewiesen, dass die Auswirkungen der Patientenmobilität auf die nationalen Gesundheitssysteme zwischen den Mitgliedstaaten beziehungsweise zwischen den Regionen eines Mitgliedstaats variieren könnten, wobei diese Unterschiede auf Faktoren wie die geografische Lage, Sprachbarrieren, das Vorhandensein von Krankenhäusern in Grenzregionen oder die Bevölkerungsgröße und das Gesundheitsbudget zurückzuführen seien.

\section{Zuständigkeiten der Mitgliedstaaten}

\section{Zuständigkeiten des Behandlungsmitgliedstaats}

Auf die in Art. 4 der Richtlinie Nr. 2011/24/EU festgelegten Zuständigkeiten des Behandlungsmitgliedstaats soll hier nur kurz eingegangen werden: Sie betreffen vor allem die Bereitstellung von einschlägigen Informationen an interessierte Patienten, die Sicherung des Bestehens von transparenten Beschwerdeverfahren und Mechanismen, damit die Patienten im Fall einer Schädigung aufgrund der erhaltenen Gesundheitsversorgung gemäß den gesetzlichen Bestimmungen des Behandlungsmitgliedstaats Rechtsbehelfe einlegen können, wie auch Systeme der Berufshaftpflichtversicherung, eine Garantie oder eine ähnliche Regelung, die im Hinblick auf ihren Zweck gleichwertig oder im Wesentlichen vergleichbar und nach Art und Umfang dem Risiko angemessen sind. Außerdem wird das Grundrecht auf Schutz der Privatsphäre bei der Verarbeitung 
personenbezogener Daten und der Anspruch des Patienten auf Erstellung einer schriftlichen oder elektronischen Patientenakte über die Behandlung und auf Zugang zu dieser Akte anerkannt und garantiert.

Von zentraler Bedeutung ist natürlich das Prinzip der Nichtdiskriminierung aus Gründen der Staatsangehörigkeit. In diesem Zusammenhang sollte die Vorschrift des Art. 4 Abs. 4 der Richtlinie Nr. 2011/24/EU gesehen werden, wonach die Mitgliedstaaten sicherstellen sollen, dass Gesundheitsdienstleister auf ihrem Hoheitsgebiet für die Behandlung von Patienten aus anderen Mitgliedstaaten die gleiche Gebührenordnung zugrunde legen, wie sie für inländische Patienten in einer vergleichbaren medizinischen Situation gilt, oder dass die in Rechnung gestellten Gebühren nach objektiven, nichtdiskriminierenden Kriterien berechnet werden, falls keine vergleichbaren Gebührensätze für inländische Patienten existieren. Nichtsdestotrotz enthält Abs. 3 den Vorbehalt, dass der Behandlungsmitgliedstaat - sofern dies durch ,zwingende Gründe des Allgemeininteresses" gerechtfertigt ist - (mehr darüber s.u.) berechtigt ist, Maßnahmen in Bezug auf den Zugang zu Behandlungen zu beschließen, um seiner grundlegenden Verantwortung gerecht zu werden, einen ausreichenden und ständigen Zugang zur Gesundheitsversorgung in seinem Hoheitsgebiet sicherzustellen. Solche Maßnahmen sind auf das notwendige und angemessene Maß zu begrenzen und dürfen kein Mittel willkürlicher Diskriminierung darstellen; ferner sind sie vorab zu veröffentlichen.

\section{Zuständigkeiten des Versicherungsmitgliedstaats}

Hinsichtlich der Zuständigkeiten des Versicherungsmitgliedstaats ist der Grundsatz der Sicherstellung der Erstattung der für die grenzüberschreitende Gesundheitsversorgung entstehenden Kosten (gemäß den Bestimmungen des Kapitels III - Art. 5 a der Richtlinie Nr. 2011/24/EU) zu berücksichtigen. Bei der "Sicherstellung der Erstattung“ geht es vor allem um die Vorbereitung, Inkraftsetzung und Sicherung der Umsetzung der entsprechenden Rechtsregulierung, die die Verhältnisse zwischen den Leistungsberechtigten und den zuständigen Trägern bestimmt. Aus den Bestimmungen der Art. 5 und 7 kann man auf keinen Fall die These ableiten, dass die Verpflichtung, die Kosten zu erstatten, gleichbedeutend ist mit der direkten Belastung des Mitgliedstaats (bzw. der Staatskasse). ${ }^{8}$

Der Versicherungsmitgliedstaat hat die Schaffung entsprechender Mechanismen sicherzustellen, um Patienten auf Anfrage Informationen über ihre Rechte und Ansprüche in diesem Mitgliedstaat im Zusammenhang mit grenzüberschreitender Gesundheitsversorgung zur Verfügung zu stellen, insbesondere bezüglich der Regeln und Bedingungen für eine Kostenerstattung. Für den Fall, dass ein Patient eine grenzüberschreitende Gesundheitsdienstleistung in Anspruch genommen hat und eine medizinische Nachbe-

8 Ausgenommen sind die Situationen, in denen der Fiskus aufgrund der geltenden Vorschriften des bürgerlichen Rechts für Schäden im Zusammenhang mit legislativer Unterlassung der Behörde haftet. 
handlung erforderlich ist, sollte für ihn dieselbe medizinische Nachbehandlung verfügbar sein, die verfügbar gewesen wäre, wenn die Gesundheitsdienstleistung im Hoheitsgebiet des Versicherungsmitgliedstaats erbracht worden wäre. Außerdem soll sichergestellt werden, dass Patienten, die grenzüberschreitende Gesundheitsversorgungsleistungen in Anspruch nehmen (möchten), mindestens eine Kopie ihrer Patientenakte haben oder per Fernabfrage darauf zugreifen können.

Nach Art. 6 der Richtlinie Nr. 2011/24/EU sind die Mitgliedstaaten verpflichtet, die Kontaktstellen für die grenzüberschreitende Gesundheitsversorgung zu benennen, die den Patienten die Informationen über die Gesundheitsdienstleister, Patientenrechte, Beschwerdeverfahren und Verfahren zur Einlegung von Rechtsbehelfen sowie über die verfügbaren rechtlichen und administrativen Möglichkeiten zur Streitbeilegung, auch bei Schäden, die im Zusammenhang mit der grenzüberschreitenden Gesundheitsversorgung entstanden sind, zur Verfügung stellen, damit die Patienten ihre Rechte im $\mathrm{Zu}-$ sammenhang mit der grenzüberschreitenden Gesundheitsversorgung wahrnehmen können.

\section{Erstattung der Kosten}

\section{Allgemeine Grundsätze - Art. 7 der Richtlinie Nr. 2011/24/EU}

Allgemeine Grundsätze der Erstattung von Kosten für die grenzüberschreitende Gesundheitsversorgung enthält Art. 7 der Richtlinie Nr. 2011/24/EU, der in Abs. 1 festlegt, dass der Versicherungsmitgliedstaat - unbeschadet der Verordnung (EG) Nr. 883/2004 und vorbehaltlich der Art. 8 und 9 - sicherstellen soll, dass die Kosten, die einem Versicherten im Zusammenhang mit einer grenzüberschreitenden Gesundheitsversorgungsleistung entstanden sind, erstattet werden, sofern die betreffende Gesundheitsdienstleistung zu den Leistungen gehört, auf die der Versicherte im Versicherungsmitgliedstaat Anspruch hat.

Die Abweichungen von dieser Grundregel sind in Abs. 2 vorgesehen, wonach ein Mitgliedstaat, wenn er in Anhang IV der Verordnung (EG) Nr. 883/2004 aufgeführt ist und gemäß jener Verordnung den Anspruch auf Leistungen bei Krankheit für Rentner und ihre Familienmitglieder, die in einem anderen Mitgliedstaat wohnen, anerkannt hat und diese Personen sich in seinem Hoheitsgebiet aufhalten, die unter die Richtlinie fallenden Gesundheitsdienstleistungen gemäß seinen Rechtsvorschriften auf eigene Rechnung erbringt, als ob die betreffenden Personen in dem Mitgliedstaat wohnen würden, der in Anhang IV aufgeführt ist. Wenn für die nach dieser Richtlinie erbrachte Gesundheitsdienstleistung keine vorherige Genehmigung erforderlich ist, wenn sie nicht gemäß Titel III Kapitel 1 der Verordnung (EG) Nr. 883/2004 erbracht wird und wenn sie im Hoheitsgebiet des Mitgliedstaats erbracht wird, der nach jener Verordnung und der Ver- 
ordnung (EG) Nr. 987/2009 letztendlich für die Kostenerstattung zuständig ist, so werden die Kosten von jenem Mitgliedstaat übernommen.

Der Versicherungsmitgliedstaat legt nach Art. 7 Abs. 3 auf lokaler, regionaler oder nationaler Ebene fest, für welche Gesundheitsversorgungsleistungen und in welcher Höhe ein Versicherter - unabhängig vom Ort der Leistungserbringung - einen Anspruch auf Kostenübernahme hat.

Nach Abs. 6 sollen für die Zwecke von Abs. 4 die Mitgliedstaaten über einen transparenten Mechanismus zur Berechnung der Kosten der grenzüberschreitenden Gesundheitsversorgung, die dem Versicherten durch den Versicherungsmitgliedstaat zu erstatten sind, verfügen. Dieser Mechanismus basiert auf vorher bekannten, objektiven und nichtdiskriminierenden Kriterien und findet auf der entsprechenden (lokalen, regionalen oder nationalen) Verwaltungsebene Anwendung. Abs. 7 bestimmt, dass der Versicherungsmitgliedstaat einem Versicherten, der einen Antrag auf Kostenerstattung im Zusammenhang mit einer grenzüberschreitenden Gesundheitsversorgung stellt, wozu auch eine Gesundheitsversorgungsleistung mit Mitteln der Telemedizin gehören kann, dieselben - auf lokaler, regionaler oder nationaler Ebene festgelegten - Voraussetzungen, Anspruchskriterien sowie Regelungs- und Verwaltungsformalitäten vorschreiben kann, die er für die gleiche Gesundheitsversorgung im eigenen Hoheitsgebiet heranziehen würde. Hierzu kann auch ein Gutachten eines Angehörigen der Gesundheitsberufe oder einer Verwaltungsstelle im Gesundheitswesen, die Leistungen für die gesetzliche Sozialversicherung oder das nationale Gesundheitssystem des Versicherungsmitgliedstaats erbringt, zählen, beispielsweise des Allgemeinmediziners oder Hausarztes, bei dem der Patient registriert ist, sofern dies für die Feststellung des individuellen Leistungsanspruchs des Patienten erforderlich ist. Dies gilt jedoch nur unter dem Vorbehalt, dass die nach diesem Absatz möglichen Voraussetzungen, Anspruchskriterien sowie Regelungsund Verwaltungsformalitäten weder diskriminierend sein noch ein Hindernis für den freien Verkehr von Patienten, Dienstleistungen oder Waren darstellen dürfen, es sei denn, es ist aufgrund des Planungsbedarfs in Zusammenhang mit dem Ziel, einen ausreichenden und ständigen Zugang zu einem ausgewogenen Angebot hochwertiger Versorgung im betreffenden Mitgliedstaat sicherzustellen, oder aufgrund des Wunsches, die Kosten zu begrenzen und nach Möglichkeit jede Verschwendung finanzieller, technischer oder personeller Ressourcen zu vermeiden, objektiv gerechtfertigt.

Von großer Bedeutung für die praktische Umsetzung der Idee der grenzüberschreitenden Gesundheitsversorgung ist die Vorschrift des Art. 7 Abs. 8 der Richtlinie Nr. 2011/24/EU. Demgemäß darf der Versicherungsmitgliedstaat die Erstattung von Kosten für die grenzüberschreitende Gesundheitsversorgung nicht von einer Vorabgenehmigung abhängig machen, mit Ausnahme der in Art. 8 genannten Fälle. Dies bedeutet grundsätzlich, dass außer der medizinischen Versorgung, die in dem abschließenden (und umstrittenen) Katalog des Art. 8 Abs. 2 der Richtlinie aufgeführt ist, die anderen Arten der grenzüberschreitenden Gesundheitsversorgung ohne Vorabgenehmigung zu- 
gänglich sein sollten, was eine wesentliche Änderung in Bezug auf die Regelung des Art. 20 der Verordnung Nr. 883/2004 darstellt.

\section{Das Recht des Versicherungsmitgliedstaats auf die Beschränkung der Anwendung}

\section{von Vorschriften über die Kostenerstattung}

In Art. 7 Abs. 9 der Richtlinie Nr. 2011/24/EU berechtigt der gemeinschaftliche Gesetzgeber die Mitgliedstaaten, gemäß der Rechtsprechung des EuGH (Abs. 12 der Präambel), die Anwendung der Vorschriften für die Kostenerstattung bei grenzüberschreitender Gesundheitsversorgung zu beschränken und zwar aus zwingenden Gründen des Allgemeininteresses, wie etwa dem Planungsbedarf in Zusammenhang mit dem Ziel, einen ausreichenden, ständigen Zugang zu einem ausgewogenen Angebot hochwertiger Versorgung im betreffenden Mitgliedstaat sicherzustellen, oder in Zusammenhang mit dem Wunsch, die Kosten zu begrenzen und nach Möglichkeit jede Verschwendung finanzieller, technischer oder personeller Ressourcen zu vermeiden. Um den Missbrauch der in dieser Vorschrift vorgesehenen Möglichkeit zu vermeiden, bestimmt Abs. 11 aber gleichzeitig, dass die Entscheidung, die Anwendung des Art. 7 gemäß Abs. 9 einzuschränken, sich auf das beschränken muss, was notwendig und angemessen ist, und dass sie keine Form der willkürlichen Diskriminierung und kein ungerechtfertigtes Hindernis für die Freizügigkeit von Personen oder den freien Verkehr von Waren oder Dienstleistungen darstellen darf. Dabei ist zu bedenken, dass die Notwendigkeit der Planung und Steuerung von Ressourcen in der Tat in jedem Gesundheitssystem, unabhängig von seiner Rechtsform, auftritt. Diese Notwendigkeit war auch immer in den vom EuGH geprüften Fällen, deren Gegenstand die Kostenerstattung von Gesundheitsversorgungsleistungen außerhalb des zuständigen Systems gewesen war, von den Parteien hervorgehoben worden. Deswegen sind die Mitgliedstaaten gemäß Art. 7 Abs. 9 der Richtlinie verpflichtet, der Kommission jede Entscheidung mitzuteilen, durch die die Erstattung von Kosten aus den in Abs. 9 genannten Gründen beschränkt wird.

Die Präambel weist in Abs. 12 darauf hin, dass der Begriff der ,zwingenden Gründe des Allgemeininteresses" vom Gerichtshof in seiner Rechtsprechung zu Art. 49 und 56 AEUV entwickelt worden sei und sich auch noch weiterentwickeln könne: Der Gerichtshof habe verschiedentlich ausgeführt, dass zwingende Gründe des Allgemeininteresses eine Einschränkung der Dienstleistungsfreiheit rechtfertigen können, wie etwa der Planungsbedarf ,in Zusammenhang mit dem Ziel, einen ausreichenden, ständigen Zugang zu einem ausgewogenen Angebot hochwertiger Versorgung im betreffenden Mitgliedstaat sicherzustellen, oder in Zusammenhang mit dem Wunsch, die Kosten zu begrenzen und nach Möglichkeit jede Verschwendung finanzieller, technischer oder personeller Ressourcen zu vermeiden“. Der Gerichtshof habe ferner anerkannt, dass auch das Ziel, eine ausgewogene, jedermann zugängliche ärztliche und klinische Versorgung aufrechtzuerhalten, insoweit unter eine der Ausnahmeregelungen aus Gründen der öffentlichen Gesundheit nach Art. 52 AEUV fallen könne, als es dazu beitrage, ein 
hohes Gesundheitsschutzniveau zu erreichen. Laut Gerichtshof erlaube diese Bestimmung des AEUV den Mitgliedstaaten, die Freiheit der Bereitstellung ärztlicher und klinischer Dienstleistungen insoweit einzuschränken, als die Erhaltung eines bestimmten Umfangs der medizinischen und pflegerischen Versorgung oder eines bestimmten $\mathrm{Ni}$ veaus der Heilkunde im Inland für die öffentliche Gesundheit erforderlich sei.

\section{Das zur Kostenerstattung verpflichtete Subjekt}

Bei den Regeln zur Kostenerstattung der grenzüberschreitenden Gesundheitsversorgung stellt sich die Frage nach dem Subjekt, das für die Rückerstattung oder Direktbezahlung verpflichtet ist. Geregelt ist dies in Art. 7 Abs. 4 der Richtlinie Nr. 2011/24/EU. Danach ist es der Versicherungsmitgliedstaat, der die Kosten der grenzüberschreitenden Gesundheitsversorgung erstattet oder direkt bezahlt, und zwar bis zu den Höchstbeträgen, die er übernommen hätte, wenn die betreffende Gesundheitsdienstleistung in seinem Hoheitsgebiet erbracht worden wäre, wobei die Höhe der tatsächlich durch die Gesundheitsversorgung entstandenen Kosten nicht überschritten werden darf. Liegen die Gesamtkosten der grenzüberschreitenden Gesundheitsversorgung über den Kosten, die bei einer Erbringung der Gesundheitsdienstleistung im Hoheitsgebiet des Versicherungsstaats übernommen worden wären, so kann der Versicherungsmitgliedstaat dennoch beschließen, die gesamten Kosten zu erstatten. Die wortlautgetreue Auslegung dieser Vorschrift, die zu dem Schluss führt, dass die Mitgliedstaaten aus ihren eigenen Mitteln die Kosten der Leistungen erstatten oder direkt bezahlen, um die grenzüberschreitende Gesundheitsversorgung bereitzustellen, ist jedoch abzulehnen. Wie schon erwähnt, ist die Vorschrift des Art. 7 Abs. 4 in Verbindung mit Art. 5 und dem allgemeinen Grundsatz aus Art. 7 Abs.1 der Richtlinie zu sehen. Danach sind die Mitgliedstaaten ganz eindeutig nur zur Sicherstellung der Erstattung oder Direktbezahlung von Kosten der grenzüberschreitenden Gesundheitsversorgung durch das zuständige Subjekt, d.h. den Träger verpflichtet. Diese Vorschrift ist aber nicht lex specialis (wie z.B. Art. 7 Abs. 2 der Richtlinie) und begründet keine Verpflichtungen der Mitgliedstaaten in Bezug auf die Finanzierung der Gesundheitsversorgung. Durch sie wird lediglich die Höhe der erstattungsfähigen Kosten begrenzt und die Kompetenz eingeräumt, die Regeln der Kostenerstattung für Patienten günstiger zu gestalten. In diesem Zusammenhang ist Art. 7 Abs. 4 S. 3 der Richtlinie zu sehen, wonach der Versicherungsmitgliedstaat beschließen kann, im Einklang mit den nationalen Rechtsvorschriften die weiteren damit verbundenen Kosten zu erstatten, wie Übernachtungs- und Reisekosten oder zusätzliche Kosten, die für Personen mit Behinderungen bei der Inanspruchnahme von grenzüberschreitenden Gesundheitsdienstleistungen infolge einer oder mehrerer Behinderungen anfallen können, sofern ausreichende Belege vorliegen, dass diese Kosten tatsächlich angefallen sind. 


\section{Gesundheitsversorgung, die einer Vorabgenehmigung unterliegen kann}

a) Abschließender Katalog der Gesundheitsversorgung mit Vorabgenehmigung

Art. 8 der Richtlinie, der die Gesundheitsversorgungsleistungen, die einer Vorabgenehmigung unterliegen können, betrifft, postuliert zunächst in Abs. 1, dass das System der Vorabgenehmigung (einschließlich der Kriterien und der Anwendung dieser Kriterien, und der Einzelentscheidungen, mit denen eine Vorabgenehmigung verweigert wird) auf das im Hinblick auf das zu erreichende Ziel notwendige und angemessene $\mathrm{Maß}$ begrenzt bleiben muss und es kein Mittel willkürlicher Diskriminierung und keine ungerechtfertigte Behinderung der Freizügigkeit der Patienten darstellen darf.

Die größte Bedeutung hat aber Abs. 2, der den abschließenden Katalog von Fällen der Gesundheitsversorgung, die einer Vorabgenehmigung unterliegen können, enthält. Dabei handelt es sich zunächst um Gesundheitsversorgung, die vom Planungsbedarf ,in Zusammenhang mit dem Ziel, einen ausreichenden, ständigen Zugang zu einem ausgewogenen Angebot hochwertiger Versorgung im betreffenden Mitgliedstaat sicherzustellen, oder in Zusammenhang mit dem Wunsch, die Kosten zu begrenzen und nach Möglichkeit jede Verschwendung finanzieller, technischer oder personeller Ressourcen zu vermeiden“, abhängig ist und eine Übernachtung des Patienten im Krankenhaus für mindestens eine Nacht oder den Einsatz einer hoch spezialisierten und kostenintensiven medizinischen Infrastruktur oder medizinischen Ausrüstung erfordert. In solchen Fällen erzeugt die Notwendigkeit der Erteilung einer Vorabgenehmigung keine Bedenken. Der gemeinschaftliche Gesetzgeber verweist dabei auf die Rechtsprechung des EuGH, auf die schon in Abs. 12, 40 und 41 der Präambel Bezug genommen wird.

Zweifelhaft sind aber zwei weitere Punkte des zweiten Absatzes. Unter Buchstabe $b$ wird die Gesundheitsversorgung aufgeführt, die „die Behandlungen mit einem besonderen Risiko für den Patienten oder die Bevölkerung einschließt" und unter Buchstabe c die Gesundheitsversorgungsleistungen, die „,von einem Erbringer von Gesundheitsdienstleistungen erbracht werden, der im Einzelfall zu ernsthaften und spezifischen Bedenken hinsichtlich der Qualität oder Sicherheit der Versorgung Anlass geben könnte, mit Ausnahme der Gesundheitsversorgung, die dem Unionsrecht über die Gewährleistung eines Mindestsicherheitsniveaus und einer Mindestqualität in der ganzen Union unterliegt".

Bezüglich der Gesundheitsversorgung ,mit einem besonderen Risiko für den Patienten oder die Bevölkerung" kann man zwar sagen, dass hier sicherlich nicht die Allgemeinmedizin in Frage kommt. Aber es ist schwer, ohne spezialisiertes Fachwissen eindeutig zu bewerten, welche Behandlung ein solches Risiko darstellen könnte. Wahrscheinlich geht es hier um die Bekämpfung von Infektionskrankheiten und Epidemien und die damit verbundenen Einschränkungen der Patientenmobilität.

Unklar ist, warum sich Punkt c nicht auf die Art der Gesundheitsversorgung, sondern auf die Versorgung bezieht, die von einem bestimmten Erbringer erbracht wird, „der im 
Einzelfall zu ernsthaften und spezifischen Bedenken hinsichtlich der Qualität oder Sicherheit der Versorgung Anlass geben könnte“. Zuerst ist fraglich, ob es hier um alle Erbringer von bestimmten Arten der Gesundheitsversorgung (z.B. Physiotherapeuten) geht, oder ob man den einzelnen Erbringer bewerten soll. Eher scheint die zweite Möglichkeit in Betracht zu kommen, umso mehr, als in der Vorschrift ausdrücklich vom „Einzelfall“ die Rede ist. Nicht ohne Bedeutung ist auch, dass die gemeinschaftlichen Vorschriften unter anderem die Anerkennung von Berufsqualifikationen vorsehen, und zwar auch in Bezug auf die Angehörigen der Gesundheitsberufe ${ }^{9}$ und dass diese Vorschriften nach Art. 2 n der Richtlinie Nr. 2011/24/EU unberührt bleiben sollen.

Angesichts dessen ist schwerlich anzunehmen, dass der Versicherungsmitgliedstaat ein klares System der Vorabgenehmigungserteilung schaffen könnte, wenn Voraussetzung die laufende Bewertung von allen einzelnen Erbringern und zwar „hinsichtlich der Qualität oder Sicherheit der Versorgung“" ist. Mit anderen Worten: Man sollte erwarten können, dass die Gesundheitsversorgung nach Art. 8 Abs. 2 der Richtlinie mit Hilfe des objektiven Kriteriums der Art der Versorgung (Krankenhausbehandlung, hochspezialisierte Behandlung oder mit einem besonderen Risiko für den Patienten oder die Bevölkerung verbundene Behandlung) definierbar und nicht auf die Bewertung der Leistung der einzelnen Erbringer bezogen wäre. Wenn dann Zweifel an der Qualität oder Sicherheit der von ihnen geleisteten Versorgung aufkommen, ist die richtige Lösung - wie auch in Art. 8 Abs. 6 der Richtlinie vorgesehen - die Verweigerung der Vorabgenehmigung auf Inanspruchnahme der grenzüberschreitenden Gesundheitsversorgung bei dem konkreten Erbringer. Die Bestimmung der vorabgenehmigungsbedürftigen Versorgung durch Verweis auf das Verhalten des Leistungserbringers „im Einzelfall“ führt in der Tat zu einer Situation, in der die Vorabgenehmigung für jede Art der Versorgung benötigt würde, weil sie von der vorherigen Beurteilung der „Qualität und Sicherheit der Versorgung" durch den Erbringer abhängig wäre. Außerdem sollen diese Kriterien im Kontext der Vorschriften des Behandlungsmitgliedstaats bewertet werden, was dem zuständigen Träger die Bewertung der Situation zusätzlich erschwert.

In diesem Lichte ist auch der Inhalt von Art. 8 Abs. 7 der Richtlinie zu verstehen, wonach der Versicherungsmitgliedstaat öffentlich zugänglich macht, welche Gesundheitsdienstleistungen einer Vorabgenehmigung im Sinne dieser Richtlinie unterliegen, und der Öffentlichkeit alle relevanten Informationen über das System der Vorabgenehmigung zur Verfügung stellt.

b) Die Beschränkung der Zulässigkeit der Verweigerung der Vorabgenehmigung

aa) Vorbehalt der Anwendung von Vorschriften der Verordnung Nr. 883/2004

Wenn es um das Verhältnis der Richtlinie zur Verordnung Nr. 883/2004 geht, ist die Bedeutung der Vorschrift des Art. 8 Abs. 3 der Richtlinie zu betonen, wonach der Ver-

9 Richtlinie 2005/36/EG des Europäischen Parlaments und des Rates vom 7. September 2005 über die Anerkennung von Berufsqualifikationen. 
sicherungsmitgliedstaat bei jedem Antrag auf Vorabgenehmigung, den ein Versicherter stellt, um eine grenzüberschreitende Gesundheitsdienstleistung in Anspruch zu nehmen, verpflichtet ist, festzustellen, ob die Bedingungen dieser Verordnung erfüllt sind und, wenn das der Fall ist, die Vorabgenehmigung gemäß der Verordnung zu erteilen, es sei denn, der Patient wünscht etwas anderes. Von wesentlicher Bedeutung bleibt natürlich „ein in Anbetracht des derzeitigen Gesundheitszustands des Versicherten und des voraussichtlichen Verlaufs seiner Krankheit medizinisch vertretbarer Zeitraum“ (Art. 20 Abs. 2 der Verordnung Nr. 883/2004). Mit anderen Worten können weder die Prozedur noch die Kriterien der Erteilung der Vorabgenehmigung für die Kostenerstattung der grenzüberschreitenden Gesundheitsversorgung, die in den zur Implementierung der Richtlinie erlassenen Vorschriften verankert werden, für den Patienten nachteiliger sein, als jene, die sich aus der Verordnungen Nr. 883/2009 und 987/2009 ableiten lassen, was ohnehin auch aus der Hierarchie der gemeinschaftlichen Rechtsakte und dem Verhältnis des gemeinschaftlichen und nationalen Rechts folgt.

bb) Der abschließende Katalog von Verweigerungsgründen (Art. 8 Abs. 6 der Richtlinie Nr. 2011/24/EU)

Für das Funktionieren des Vorabgenehmigungssystems der Inanspruchnahme von Leistungen der grenzüberschreitenden Gesundheitsversorgung wird die Regelung, die deren eventuelle Verweigerung betrifft, von wesentlicher Bedeutung sein. Die Richtlinie bestimmt insbesondere, dass der Versicherungsmitgliedstaat (vorbehaltlich der schon erwähnten Vorschrift des Art. 8 Abs. 6) eine Vorabgenehmigung nicht verweigern darf, wenn der Patient nach Art. 7 Anspruch auf die betreffende Gesundheitsversorgung hat und die betreffende Gesundheitsversorgung nicht auf seinem Hoheitsgebiet innerhalb eines medizinisch vertretbaren Zeitraums geleistet werden kann - unter Berücksichtigung einer objektiven medizinischen Beurteilung des Gesundheitszustands des Patienten, der Vorgeschichte und der voraussichtlichen Entwicklung der Krankheit, des Ausmaßes der Schmerzen und/oder der Art der Behinderung des Patienten zum Zeitpunkt der erstmaligen oder erneuten Beantragung der Genehmigung. Diese Vorschrift ist in der Tat eine Wiederholung des Art. 20 der Verordnung Nr. 883/2004 und knüpft an die Rechtsprechung des EuGH an, die noch aufgrund des Art. 22 der Verordnung Nr. 1408/71 entstanden ist.

In diesem wird die Vorschrift des Art. 8 Abs. 6 der Richtlinie Nr. 2011/24/EU relevant, die einen abschließenden Katalog von Verweigerungsgründen enthält.

Die Verweigerung ist danach vor allem dann begründet, wenn der Patient nach klinischer Bewertung mit hinreichender Sicherheit einem nicht annehmbaren Sicherheitsrisiko ausgesetzt wird, wobei der potenzielle Nutzen der gewünschten grenzüberschreitenden Gesundheitsversorgung für den Patienten berücksichtigt wird. Es geht also um die objektiven medizinischen Kriterien, die z.B. mit den Risiken des Patiententransports verbunden sind.

Als weiterer Verweigerungsgrund gilt der Fall, dass die Öffentlichkeit mit hinreichender Sicherheit einem erheblichen Sicherheitsrisiko infolge der betreffenden grenz- 
überschreitenden Gesundheitsversorgung ausgesetzt wird. Wie schon oben erwähnt, ist es schwer, sich ohne entsprechendes Fachwissen eine andere Situation als eine epidemiologische Gefährdung vorzustellen, in der die Inanspruchnahme von Leistungen der grenzüberschreitenden Gesundheitsversorgung durch einen bestimmten Berechtigten die Sicherheit der Öffentlichkeit bedrohen könnte.

Fragwürdig ist aber auch der unter Buchstabe c genannte Verweigerungsgrund, wonach die Vorabgenehmigung dann ebenfalls versagt werden kann, wenn die beantragte Gesundheitsversorgungsleistung von einem Erbringer geleistet werden soll, der zu ernsthaften und spezifischen Bedenken in Bezug auf die Einhaltung der Qualitätsstandards und -leitlinien für die Versorgung und die Patientensicherheit einschließlich der Bestimmungen über die Überwachung, Anlass gibt, ungeachtet der Tatsache, ob diese Standards und Leitlinien in Rechts- und Verwaltungsvorschriften oder durch vom Behandlungsmitgliedstaat eingerichtete Akkreditierungssysteme festgelegt sind. Wenn der zuständige Träger seine Ablehnung der Vorabgenehmigung auf diesen Grund stützen möchte, sollte er die Anträge von Berechtigten in der Tat nicht nur im Lichte der Art der beantragten Versorgung (Art. 8 Abs. 7 der Richtlinie), sondern auch in Bezug auf den individualisierten Erbringer und die Art und Weise seiner Leistung bewerten. Dies wiederum kann sich in der Praxis nicht nur als schwere und mühsame, sondern auch als was für den interessierten Berechtigten viel wichtiger ist - zeitaufwendige Aufgabe erweisen.

Schließlich bestimmt die Richtlinie den Fall zum Verweigerungsgrund, dass die betreffende Gesundheitsversorgung unter Berücksichtigung des gegenwärtigen Gesundheitszustands und des voraussichtlichen Krankheitsverlaufs des jeweils betroffenen Patienten auf seinem Hoheitsgebiet innerhalb eines medizinisch vertretbaren Zeitraums geleistet werden kann. Angesichts Art. 8 Abs. 5 der Richtlinie und Art. 20 der Verordnung Nr. 883/2004 kann man sagen, dass dieser Vorbehalt eine unnötige Wiederholung dieser Vorschriften ist.

\section{Schlussbemerkungen}

Es wird sicherlich noch eine gewisse Zeit dauern, bis wir die Möglichkeit haben, die Implementierungsvorschriften bewerten zu können. Aufgrund der Präambel und des normativen Teils der Richtlinie Nr. 2011/24/EU scheint aber die Erwartung begründet, dass sich in der Tat die rechtliche Situation von berechtigten Patienten, die Leistungen der grenzüberschreitenden Gesundheitsversorgung in Anspruch nehmen möchten, nicht bedeutend ändern wird. In Bezug auf Krankenhaus- und spezialisierte Behandlungen bleibt der „Planungsbedarf“ ein Argument dafür, den Zugang außerhalb des zuständigen Systems von der Vorabgenehmigung abhängig zu machen, wobei die Wartezeit auf die 
konkrete Leistung, bewertet vor dem Hintergrund des Gesundheitszustands des Patienten, das Hauptkriterium sein soll.

Die Analyse der Vorschriften der Verordnung und der Richtlinie berechtigt auch zu der Feststellung, dass die grenzüberschreitende Mobilität von Patienten, d.h. von Berechtigten sich vor allem auf die Leistungen der allgemeinen Gesundheitsversorgung auf der regionalen und lokalen Ebene, also in den Grenzgebieten, beschränkt. Dies scheint ganz im Sinne von Abs. 4 der Präambel, wonach die Patienten bei der Umsetzung der Richtlinie in einzelstaatliche Rechtsvorschriften und bei deren Anwendung nicht dazu ermuntert werden sollten, Behandlungen in einem anderen als ihrem Versicherungsmitgliedstaat in Anspruch zu nehmen. 


\title{
Auslandstätigkeiten und das Recht auf polnische Überbrückungsrenten
}

\author{
Marcin Zieleniecki
}

A. Die historische Entwicklung des Rechts auf vorzeitige Pensionierung infolge besonderer Arbeitsbedingungen

B. Reformbestrebungen

C. Die Einführung der Überbrückungsrente

D. Die Berücksichtigung von Auslandstätigkeiten

E. Die Etablierung eines Systems zum elektronischen Datenaustausch

A. Die historische Entwicklung des Rechts auf vorzeitige Pensionierung infolge besonderer Arbeitsbedingungen

Seit Mitte der fünfziger Jahre des 20. Jahrhunderts können unter besonderen Bedingungen beschäftigte Arbeitnehmer das im polnischen Sozialversicherungssystem verankerte Recht auf Pensionierung vor Erreichen des allgemein vorgeschriebenen Pensionierungsalters nutzen. Voraussetzung dafür ist, dass die ausgeführte Tätigkeit in einem beträchtlichen Grad gesundheitsschädlich oder beschwerlich ist oder hohe psychischkörperliche Ansprüche stellt. Dem trägt die Verordnung vom 25. Juni 1954 über die allgemeine Altersversorgung von Arbeitnehmern und deren Familien ${ }^{1}$ Rechnung, die bei zur so genannten Kategorie I gehörenden Arbeitnehmern die Berechtigung auf Altersrente für Frauen im Alter von 55 Jahren und für Männer im Alter von 60 Jahren vorsieht. Jedoch setzt dies voraus, dass der gesamte Beschäftigungszeitraum 20 Jahre (für Frauen) bzw. 25 Jahre (für Männer) beträgt und davon zumindest 15 Jahre unter Bedingungen der Kategorie I gearbeitet wurde. Begleitet wurde dies von der Anwendung günstigerer Berechnungsgrundsätze im Bereich der Altersversorgung, der Invalidenrente und der Gewährung von Zuschüssen. Ähnliche Modelle wurden im genannten Zeit-

1 GBl. der Republik Polen Nr. 30, Pos. 116. 